\title{
Evaluation of FunFRIENDS program in prevention of anxiety in Brazilian children: a randomized controlled pilot trial
}

\author{
Avaliação do programa FunFRIENDS na prevenção da ansiedade \\ em crianças brasileiras: um estudo piloto randomizado controlado
}

Lina Maria Herrera Nunez Rivero (https://orcid.org/0000-0001-7891-7212) ${ }^{1}$

André Luiz Monezi Andrade (https://orcid.org/0000-0003-0111-8935) ${ }^{2}$

Larissa Zeggio Perez Figueredo (https://orcid.org/0000-0003-4150-1584) ${ }^{3}$

Bruno de Oliveira Pinheiro (https://orcid.org/0000-0002-3671-3171) ${ }^{1}$

Denise De Micheli (https://orcid.org/0000-0001-8546-4354) ${ }^{1}$
${ }^{1}$ Departamento de Psicobiologia, Universidade Federal de São Paulo. São

Paulo SP Brasil.

${ }^{2}$ Centro de Ciências

da Vida, Pontifícia

Universidade Católica

de Campinas. Av. John

Boyd Dunlop s/n, Jardim

Ipaussarama. 13034-685

Campinas SP Brasil.

andre.andrade@

puc-campinas.edu.br

${ }^{3}$ Instituto Brasileiro de

Inteligência Emocional e

Social. São Paulo SP Brasil.

\begin{abstract}
This study aimed to evaluate the effects of the FunFRIENDS protocol on anxiety symptoms, internalizing problems and prosocial behavior in 4- to 5-year old children. Participated of this study 43 children with an allocation ratio of 1:1 between groups (Intervention Group [IG], $N=21$; Control Group [CG], $N=22$ ) from a public school in the city of Sao Paulo participated in the study. Behaviors were evaluated by using standardized questionnaires (PAS, CBCL, SDQ, $S C B E)$ assessing the behavioral profile of the children at three different periods; baseline $\left(T_{0}\right)$, one month $\left(T_{1}\right)$ and three months $\left(T_{2}\right)$ after the intervention. It was observed a reduction in the intensity of anxiety symptoms in the IG after the intervention but with no significant effect. Overall, there were no significant differences between the $I G$ and the CG in most behaviors on all periods evaluated. On both Intention-to-Treat analyses also it was not detected significant effects of the intervention. This is the first Brazilian study evaluating the effects of FunFRIENDS program and the implications of these findings are discussed and its significance for future research.
\end{abstract}

Key words Anxiety, Cognitive therapy, Health promotion, Primary prevention
Resumo Este estudo teve como objetivo avaliar os efeitos do protocolo FunFRIENDS sobre sintomas de ansiedade, problemas de internalização e comportamento pró-social em crianças de 4 a 5 anos de idade. Participaram 43 crianças que foram alocadas na proporção de 1:1 entre os grupos: (Grupo Intervenção [GI], $N=21$; Grupo Controle [GC], $N=22$ ) de uma escola pública da cidade de São Paulo. Os comportamentos foram avaliados por meio de questionários padronizados (PAS, CBCL, SDQ, SCBE), que avaliaram o perfil comportamental das crianças em três momentos distintos; linha de base $\left(T_{0}\right)$, um mês $\left(T_{1}\right)$ e três meses $\left(T_{2}\right)$ após a intervenção. Observou-se uma redução na intensidade dos sintomas de ansiedade no GI após a intervenção, mas sem efeito significativo. De forma geral, não houve diferenças significativas entre o GI e o GC na maioria dos comportamentos em todos os períodos avaliados. Nas duas análises de Intenção-de-Tratar, também não foram detectados efeitos significantes da intervenção. Este é o primeiro estudo no Brasil avaliando os efeitos do programa FunFRIENDS e as implicações destes achados são discutidas para futuras pesquisas.

Palavras-chave Ansiedade, Terapia cognitiva, Promoção da saúde, Prevenção primária 


\section{Introduction}

Anxiety is one of the most common and treatable mental illness in children and adolescents, with a global prevalence ranging from $4 \%$ to $25 \%^{1}$. In a study with 1,251 Brazilians between 7 and 14 years of age, $10.5 \%$ showed high anxiety levels, $4.4 \%$ being boys and $6.1 \%$ girls ${ }^{2}$. In a recent report by the World Health Organization ${ }^{3}$, Brazil was the country with the highest prevalence of anxiety disorders $(8.3 \%)$ and ranks fifth in the prevalence of depressive disorders $(5.8 \%)$. Concerning children under five years, some authors have detected an increase in the incidence of anxiety disorders, especially over the last decade ${ }^{4}$. This clinical condition may worsen over time and have severe effects, especially among those children who have not received appropriate support $^{5}$, bringing impairments in the short, medium and long term such as low self-esteem, school drop-out, substance misuse, and psychological problems.

Human development is influenced by biological, cognitive, affective and social dimensions and throughout the life cycle diverse contexts interact, such as family and school, and when these connections are positive, they can promote processes of resilience and result in a better quality of life. Each of these contexts may offer protective or risk factors, and the school environment has a key role in children's socialization because it is the place where children spend most of their time $^{7}$. In this context, children experience different situations: peer relationships, groups, friendship, competition, learning and discovery of new, becoming the main environment where psychological suffering in childhood can be screened ${ }^{5,8}$. Thus it is important to screen and develop different intervention strategies for school environments. Programs focused on the prevention of mental illness are one of the broadest types of psychological intervention for school children, with one of the most common of these intervention strategies being the promotion of protective mental health factors, such as the development of social-emotional skills?.

In this sense, some prevention programs have been developed in many countries, such as the FRIENDS program, which is focused on children and adolescents and is based on Cognitive Behavioural Therapy ${ }^{10}$. The main components of FRIENDS are focused on reducing symptoms of anxiety through the development and reinforcement of social-emotional skills. The word friends is an acronym that describes the skills taught in the program ( $F$ - Feelings; $R$ - Relax; $I$ - I can try; $E$ - Encourage; $N$ - Nurture; $D$ - Don't forget; $S$ - Stay brave). The original version of the FRIENDS program was initially proposed for schoolchildren from 7 to 14 years of age ${ }^{10}$, and its effectiveness was evaluated in different countries and cultures with good results ${ }^{11,12}$.

An adaptation of FRIENDS was developed by Pahl and Barrett ${ }^{13}$ for children between the ages of 4 to 7 and was called FunFRIENDS. The authors evaluated the efficacy of the intervention in 263 Australian children aged 4 to 6 . Although testing showed improvements in both the intervention and a control group immediately following the intervention, the intervention group had lower levels of anxiety and social inhibition at the 12-month follow-up compared to the control group.

Although some studies have evaluated the effectiveness of FunFRIENDS in several coun$\operatorname{tries}^{14,15}$ as yet, there has been no research on the use of this intervention in Brazilian children. The objective of this study was to evaluate the effectiveness of the FunFRIENDS program in reducing the symptoms of anxiety in children aged 4 to 7 . We also evaluated the effects of this intervention on the prosocial behavior. We hypothesized that the intervention group would have lower levels of anxiety and depression and a higher frequency of prosocial behaviors after the intervention than the control group. Also, levels of anxiety and depression would remain significantly lower and the frequency of prosocial behaviors significantly higher during the follow-up period compared to children in the control group.

\section{Method}

\section{Study Design}

The study is a randomized controlled pilot study with an allocation ratio of 1:1 between the experimental and control groups. The equivalence between groups at the baseline was analyzed using the one-way analysis of variance (ANOVA) for the continuous variables, and the chi-square test was used for the nominal variables. No significant differences were detected between the groups $\left(\mathrm{T}_{0}\right)$ for any of the studied variables.

\section{Participants}

We performed a randomized controlled pilot study with 43 pre-school children $\left(\mathrm{M}_{\text {age }}=4.93\right.$, 
$\mathrm{SD}=0.33, \mathrm{Md}_{\text {age }}=5$, min-max $\left.=4-6\right)$ from a school in the city of São Paulo. The children were randomly assigned to the Intervention Group (IG) or the Control Group (CG), with 21 children in the IG $\left(\mathrm{M}_{\text {age }}=4.85, \mathrm{SD}=0.35, \mathrm{Md}_{\text {age }}=\right.$ 5 , min-max $=4-5,13$ girls, 8 boys) and 22 children in the CG $\left(\mathrm{M}_{\text {age }}=5, \mathrm{SD}=0.30, \mathrm{Md}_{\text {age }}=5\right.$, $\min -\max =4-6,10$ girls, 12 boys). The inclusion criteria were: children regularly attending school, aged between 4 and 7 years of age, and parental consent and agreement of the school to participate in the study. Figure 1 is a flowchart of participation at different stages of the study, according to the guidelines of the Consolidated Standards of Reporting (CONSORT) trials.

\section{FunFRIENDS intervention design}

The FunFRIENDS program was delivered through 14 sessions with durations of between 90 and 120 minutes each, being: a) 10 weekly sessions for group activities with children; b) 2 sessions with parents/guardians, teachers and members of the community; c) 2 behavior reinforcement sessions: the first session was held one month after the $10^{\text {th }}$ meeting and the second three months after the 10th meeting. Two psychologists conducted all intervention activities with specific training in this protocol.

The content of the sessions were distributed as follows: 1) Presentation of the program to the participant and relationship building; 2) Discussion about the normalization of emotions, identification of emotions in themselves and others; 3) Identification of emotions in themselves and others (postural, facial and meta-verbal expression); 4) Introduction of techniques of emotional self-regulation; 5) Introduction to the concept of the relationship between thoughts, feelings, and behavior; 6) Identification of thoughts that help and do not help, replacement of thoughts; 7) Presentation of the concept of a step-by-step coping plan; 8) Step-by-step coping plan training; 9) Introduction to self-reward and support network; 10) Techniques of positive attention, self-confidence, revision of the program; 11) First session to reinforce the skills learned; 12) the Second session of reinforcement and closure of the program with "graduation" and picnic.

\section{Instruments}

The Preschool Anxiety Scale (PAS) was used to evaluate six dimensions of anxiety symptoms (Separation Anxiety Subscale; Social Anxiety
Subscale; Obsessive Compulsive Subscale; Physical Injury Fears; Generalized Anxiety; Total anxiety) from 28 items rated on a 5-point Likert scale, with 0 being "never" to 4 being "always". The instrument had previously been adapted and validated for Brazilian Portuguese ${ }^{16}$ and had been shown to have high internal consistency $(\alpha=$ $0.88)$. A T-score of 60 as indicative of sub-clinical or elevated levels of anxiety.

The Child Behavior Checklist (CBCL $1^{1 / 2}$ 5) was used to evaluate externalizing problems (aggressiveness and attention problems) and internalizing (emotional reactivity, depression/ anxiety, somatic complaints, and withdrawal). The instrument has 99 items scored from 0 to 2 , based on the incidence of the behaviors observed by the caregiver. The total of all items composes the raw score, with the possibility of change from zero to 200 . Thus, the score for behavioral problems provides the child's behavior profile. Thus, the results are obtained regarding a $\mathrm{T}$ score, which is a score ranging from 50 to 100 , standardized according to the sex and age of the child, based on the American population. These scores determined the following categories: non-clinical $(\leq 51$ percentile $93, \mathrm{~T}$ score $\leq$ 60 ), borderline (from the 94th to 97th percentile, $\mathrm{T}$ score between 60 and 63$)$ and clinical $(\geq 97 \mathrm{th}$ percentile, $\mathrm{T}$ score $\geq 64$ ), for the behavioral scales of CBCL $1 \frac{1}{2}-5$. Noting that the scores of the scales are not equivalent to a diagnosis, refer to the effect of the problem rather than its duration. The instrument had been previously adapted and validated for Brazilian Portuguese ${ }^{17}$ and had been shown to have a high overall internal consistency $(\alpha=0.97)$.

The Strengths and Difficulties Questionnaire (SDQ) consisted of 25 items and was used to assess emotional symptoms, conduct problems, hyperactivity, peer relationship problems, and prosocial behavior. For each of the five subscales, scores can range from 0 to 10 , being the total score of difficulties generated by the sum of the results of all subscales except sociability, ranging from 0 to 40 points. The cut-off score for the total score of difficulties was 17 points. The instrument had previously been adapted and validated for Portuguese ${ }^{18}$ and had been shown to have high internal consistency $(\alpha=0.80)$.

The Social Competence and Behavior Evaluation Scale (SCBE) was applied only among teachers to evaluate the social adaptation of children in the school environment. It consists of 30 items that assess social competence, regulation, and expression of emotion, anger and aggressive 


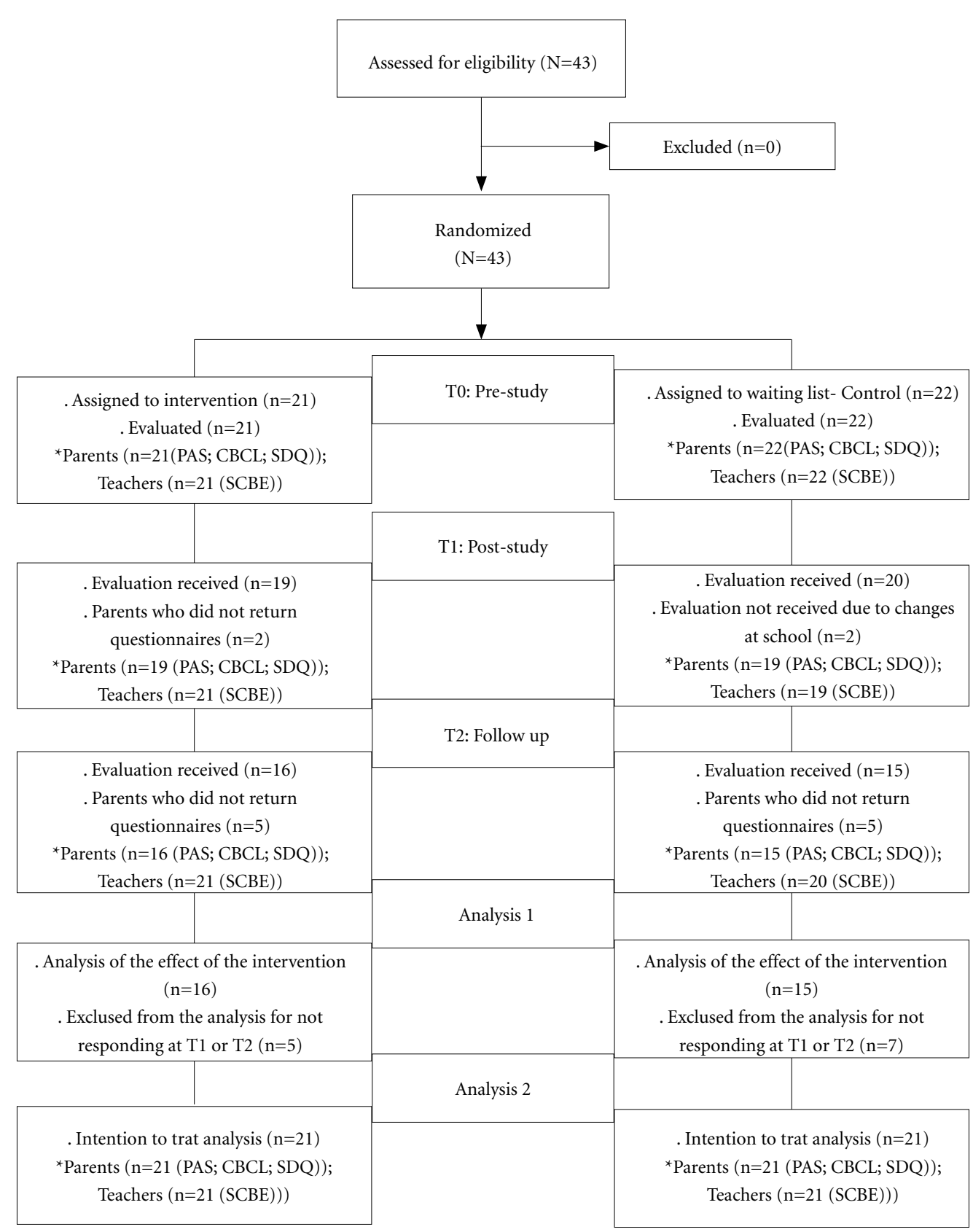

Figure 1. Flow diagram of the participants of the study (randomized sample) according to CONSORT guidelines.

behaviors, and anxiety and avoidance behaviors. Items are rated on an incidence scale of 1 (almost never happens) to 6 (almost always). The instrument had previously been adapted and validated for Portuguese ${ }^{19}$ and had been shown to have high overall internal consistency $(\alpha=0.87)$.

\section{Procedures}

After the study was approved by the Regional Board of Education of the city of Sao Paulo, three meetings were held, the first with the school teachers and the others with the parents of the 
students selected to take part in the study. The main subjects discussed were: emotional health, anxiety, depression and childhood resilience. The first data collection $\left(\mathrm{T}_{0}\right)$ took place following the meetings, with parents/caregivers who signed a free and informed consent form and then completed the questionnaires. The same procedure was carried out with the teachers.

During the intervention, the IG also received a FunFRIENDS activity book aimed at reinforcing the skills they had learned and practiced in each meeting. Also, the parents received a weekly report detailing the skills developed in each session and giving instructions on the activities to be performed at home. Between the 5th and 6th application of the FunFRIENDS program, the parents of the IG children were invited to a second 60-minute presentation on strategies for maintaining resilience in the family environment, building healthy habits, parental strategies, and social-emotional skills. The parents and teachers of all the children were again invited to complete the instruments at the end $\left(T_{1}\right)$ of the intervention (10th week), and three months after its completion $\left(\mathrm{T}_{2}\right)$.

After the $\mathrm{T}_{2}$, the children in the control group were submitted to the intervention with the same experimental conditions described in this study.

\section{Ethics}

The present study was approved by the Research Ethics Committee of the Federal University of São Paulo (UNIFESP) and was registered at the Brazilian Registry of Clinical Trials.

\section{Data analysis}

Data from the continuous variables were standardized using the Z-score method to identify possible data outliers $(-3 \leq Z$-Score $\geq 3)$ that could affect the data analysis ${ }^{20,21}$. A chi-square test was used for analysis of categorical variables, and a one-way analysis of variance for continuous variables. Two-way mixed ANOVA with repeated measures was used to evaluate the intervention, with the different behavioral dimensions evaluated by the instruments as dependent variables. The factors "time" $\left(\mathrm{T}_{0}, \mathrm{~T}_{1}\right.$, and $\left.\mathrm{T}_{2}\right)$ and "group" (IG and CG) were considered as independent variables. When significant effects were detected, Newman-Keuls post-hoc test was performed.

Additional analyses were carried out using an Intention to Treat (ITT) model based on two specific approaches used in the previous studies ${ }^{20}$. In the first one (ITT-1), the last-observation-carried-forward (LOCF) procedure was used with those children who did not complete the intervention having their baseline values $\left(\mathrm{T}_{0}\right)$ repeated at both $\mathrm{T}_{1}$ and $\mathrm{T}_{2}$. Thus, four participants ( $n=$ 2 IG, $n=2$ CG) had their values repeated after $\mathrm{T}_{1}$, 12 participants at $\mathrm{T}_{2}(n=5 \mathrm{IG}, n=7 \mathrm{CG})$. In the second protocol (ITT-2), the mean values of the participants who completed the intervention at $\mathrm{T}_{1}(n=20)$ and $\mathrm{T}_{2}(n=19)$ from their respective groups (IG and CG) were considered.

The size of the effect of the nominal variables was evaluated using Cramer's V Test, from the following degrees of freedom; $d f=1$ ( 0 to $0.1=$ small effect), $(0.11$ to $0.3=$ medium effect $),(0.31$ to $1.0=$ large effect $) ; d f=2(0$ to $0.07=$ small effect $),(0.08$ to $0.21=$ medium effect $),(0.22$ to $1.0=$ large effect). For the analysis of variance, the Eta Square Test ( $\eta 2$ ) was used considering the following values: ( 0 to $0.4=$ small effect, 0.41 to $0.79=$ medium effect, 0.8 to $1.0=$ large effect $)^{22}$. The level of significance in all analyses was $5 \%$, and the software used was IBM SPSS version 20 .

We used the "JT Method" to better evaluate the reliability changes of scores before and after the intervention. This method was developed by Jacobson and $\operatorname{Truax}^{23}$ and allows evaluating the clinical relevance of the behaviors change of each participant. Due to this fact, JT Method is indicated to a small sample size because indicates if the behavioral changes detected during the intervention were a consequence of the intervention or due to measurements errors. The cut point of the scores (clinical or non-clinical group) was based on the normative data of SDQ instrument available by the authors who adapted and validated the instruments in Brazil as described above ${ }^{18}$. To perform the analyses and the graphs we used the following site ${ }^{24}$ : http://www.psicoinfo.ufscar. br/conteudo/o-metodo-jt

\section{Results}

As shown in Table 1, at the beginning of the intervention $\left(\mathrm{T}_{0}\right)$ ANOVA did not detect differences in age between participants in the intervention group $(\mathrm{M}=4.7, \mathrm{SD}=0.47)$ and the control group $(\mathrm{M}=4.9, \mathrm{SD}=0.25)$. There were also no significant differences in the distribution of boys and girls in the two groups studied, and ANOVA did not detect differences between the groups for any of the symptoms and behaviors analyzed. When the effects of the intervention were evaluated from the different instruments, ANOVA did 
Table 1. Evaluation of the behavior of the children from both groups regarding the perception of parents and teachers at three different times $\left(\mathrm{T}_{0}, \mathrm{~T}_{1}\right.$ and $\left.\mathrm{T}_{2}\right)$.

\begin{tabular}{|c|c|c|c|c|c|c|c|c|c|c|c|c|c|c|c|}
\hline & \multicolumn{6}{|c|}{ Intervention Group } & \multicolumn{6}{|c|}{ Control Group } & \multirow{3}{*}{ Test $\mathrm{F}$} & \multirow{3}{*}{$\mathbf{p}$} & \multirow{3}{*}{ Effect } \\
\hline & \multicolumn{2}{|c|}{$\mathrm{T}_{0}$} & \multicolumn{2}{|c|}{$T_{1}$} & \multicolumn{2}{|c|}{$\mathrm{T}_{2}$} & \multicolumn{2}{|c|}{$\mathrm{T}_{0}$} & \multicolumn{2}{|c|}{$T_{1}$} & \multicolumn{2}{|c|}{$\mathrm{T}_{2}$} & & & \\
\hline & $\mathbf{M}$ & SD & $\mathbf{M}$ & SD & $\mathbf{M}$ & SD & $\mathbf{M}$ & SD & $\mathbf{M}$ & SD & $\mathbf{M}$ & SD & & & \\
\hline \multicolumn{16}{|l|}{ PAS } \\
\hline Social anxiety & 8.50 & 3.76 & 8.25 & 4.91 & 6.87 & 4.37 & 9.93 & 3.56 & 8.60 & 3.99 & 8.13 & 3.31 & 0.26 & 0.77 & 0.01 \\
\hline Separation anxiety & 8.73 & 2.93 & 7.86 & 2.69 & 5.33 & 1.63 & 8.50 & 4.63 & 6.68 & 4.37 & 5.68 & 3.73 & 0.40 & 0.67 & 0.02 \\
\hline Total scores & 41.18 & 16.86 & 36.62 & 17.25 & 31.0 & 17.19 & 40.93 & 12.26 & 33.40 & 11.04 & 30.30 & 9.50 & 0.11 & 0.89 & 0.00 \\
\hline \multicolumn{16}{|l|}{ SDQ } \\
\hline Emotional symptoms & 3.18 & 2.04 & 2.50 & 2.03 & 2.25 & 1.84 & 2.20 & 1.87 & 1.80 & 1.32 & 1.53 & 1.18 & 0.34 & 0.67 & 0.01 \\
\hline Peer Problems & 2.37 & 1.58 & 2.87 & 1.92 & 1.87 & 1.82 & 1.86 & 1.59 & 1.66 & 1.54 & 1.33 & 1.29 & 0.94 & 0.40 & 0.03 \\
\hline Prosocial behavior & 7.75 & 2.14 & 7.75 & 2.17 & 7.75 & 1.94 & 8.0 & 1.69 & 8.20 & 1.97 & 7.80 & 1.37 & 0.26 & 0.77 & 0.01 \\
\hline Total scores & 11.13 & 4.89 & 9.60 & 4.13 & 8.93 & 4.92 & 14.0 & 5.31 & 14.0 & 5.37 & 11.87 & 5.48 & 0.92 & 0.41 & 0.03 \\
\hline \multicolumn{16}{|l|}{ CBCL $\left(1 \frac{1 / 2}{2} 5\right)$} \\
\hline Anxiety/depression & 4.62 & 2.68 & 4.56 & 2.42 & 3.75 & 2.35 & 4.06 & 2.76 & 3.60 & 1.91 & 3.13 & 1.18 & 0.12 & 0.89 & 0.00 \\
\hline Somatic symptoms & 2.06 & 2.51 & 2.06 & 2.37 & 2.18 & 2.22 & 2.06 & 1.62 & 1.60 & 1.35 & 1.33 & 1.29 & 0.69 & 0.51 & 0.03 \\
\hline Internalizing symptoms & 14.31 & 8.03 & 13.56 & 7.81 & 13.43 & 7.42 & 11.0 & 6.95 & 10.0 & 5.89 & 8.33 & 4.41 & 0.48 & 0.62 & 0.02 \\
\hline Anxiety problems & 6.06 & 3.64 & 5.50 & 3.2 & 4.68 & 3.60 & 5.60 & 2.99 & 4.86 & 2.19 & 3.73 & 2.43 & 0.28 & 0.76 & 0.01 \\
\hline Affective problems & 4.43 & 3.16 & 3.87 & 2.47 & 4.12 & 2.75 & 2.80 & 2.11 & 3.33 & 2.76 & 2.06 & 2.08 & 3.15 & 0.05 & 0.10 \\
\hline Total scores & 51.12 & 24.3 & 44.87 & 21.23 & 45.87 & 21.60 & 37.4 & 18.89 & 36.53 & 18.46 & 28.4 & 16.09 & 2.67 & 0.08 & 0.09 \\
\hline \multicolumn{16}{|l|}{ SCBE } \\
\hline Anxiety & 1.48 & 0.58 & 1.62 & 0.65 & 1.54 & 0.57 & 1.50 & 0.56 & 1.36 & 0.47 & 1.24 & 0.36 & 1.46 & 0.24 & 0.05 \\
\hline Prosocial behaviors & 4.65 & 1.60 & 4.01 & 1.30 & 5.04 & 1.17 & 4.24 & 0.89 & 3.78 & 0.74 & 4.68 & 0.70 & 0.08 & 0.89 & 0.00 \\
\hline
\end{tabular}

Note: PAS = The Preschool Anxiety Scale; SDQ = The Strength and Difficulties Questionnaire; CBCL $=$ The Child Behaviour Check List 1 1/2/2 5 years; SCBE = The Social Competence and Behavior Evaluation Scale; SD =

Standard deviation; IG = Intervention group; $\mathrm{CG}=$ Control group. 
not detect differences in the factor group (IG and CG) and time $\left(\mathrm{T}_{0}, \mathrm{~T}_{1}\right.$ e $\left.\mathrm{T}_{2}\right)$ in any of the studied dimensions. Regarding the PAS data, there was a reduction in social anxiety levels between $\mathrm{T}_{0}$ and $\mathrm{T}_{2}$ in both groups, especially in the IG participants but this reduction was not significant.

When data were assessed from the ITT-1 protocol (Table 2), a reduction in separation anxiety levels and overall scores in both groups was observed for the PAS instrument, but without statistical significance.

About the ITT-2 protocol (Table 3), the participants in the IG group also presented a more significant decrease in social anxiety scores in the PAS, and a lower overall SDQ score, although without statistical significance for both instruments

The Figure 2 shows all participants from IG (bullets) and those bullets upper the horizon line indicates adjusted behaviors measured by SDQ instrument. Regarding the participants S4 and S6, it was observed they passed from misadjusted to adjusted behaviors due to the intervention. Those participants from the second quadrant (S1, S2, S3, S5, S7, S9, S10, S11, S12, S13 and S14) remained with adjusted behaviors whereas those from the third quadrant (S8, S15 and S18) remained on the misadjusted after the intervention.

\section{Discussion}

To the best of our knowledge, this is the first Brazilian study conducted to evaluate the effects of a universal preventative intervention in schools through the program FunFRIENDS. The main results did not indicate significant effects in the intervention group in any of the dimensions studied. A small reduction in social anxiety levels was detected in the children of both groups and JT analysis indicated that only two children (S4 and S6) showed improvement of their behaviours according the SDQ instrument.

These findings corroborate some studies in the literature, such as the report of Pahl and Barrett $^{13}$ in which the authors did not detect a reduction in anxiety levels in IC immediately after the end of the program. On the other hand, Barrett et al. ${ }^{25}$ observed a significant reduction in anxiety symptoms in an intervention group of children aged 5 to 7 years evaluated using the PAS and BIQ (Behavioural Inhibition Questionnaire), with the results being maintained at 12-month follow-up.

It is possible that the heterogeneity of these findings is associated with the different instru- ments used. In the study by Pahl and Barrett ${ }^{13}$, it was detected a decrease in anxiety levels in the intervention group only from the teachers' report (BIQT instrument). In Dohl's study ${ }^{26}$, anxiety reduction was significantly higher in the intervention group and was maintained after 12 months according to the PAS and SDQ adapted for teachers. However, the same instruments were used by parents and no significant effect of the intervention was identified. In Mexico, Zertuche ${ }^{14}$ observed a small reduction in anxiety symptoms, but with no statistical difference, using the PAS scale completed by parents of preschool children. However, when the BIQ scale was used a statistically significant reduction was observed in anxiety symptoms. On the other hand, in a study by Barret et al. ${ }^{25}$, the authors evaluated anxiety from parent-directed instruments (PAS and BIQ).

The PAS scale, therefore, may not be the most appropriate for the evaluation of anxiety symptoms in preschool children, mainly when the intervention is universal and carried out in a school environment. Future research using FunFRIENDS with Brazilian pre-schoolers could use other, more sensitive instruments - as seems to be the case with the BIQ scale - to clarify differences in the results of the effects of programs on anxiety symptoms.

Although anxiety is one of the main components of internalizing problems, sadness, withdrawal, somatic complaints and fear are also important indicators and are examined together in several studies ${ }^{27}$. In the present study, the instruments used also evaluated this broader category of internalizing symptoms and found no reductions in these symptoms in preschool children with the CBCL instrument.

Shortt et al. ${ }^{28}$ conducted a randomized study with a clinical sample of children aged 6 to 10 years old using the CBCL scale completed by the mothers and found that the FunFRIENDS intervention had a significant effect on the reduction of internalizing symptoms among children in the intervention group. A similar result was found in a study conducted with children aged 7 to 10 years in school settings ${ }^{29}$. Similarly, Gallegos-Guajardo et al. ${ }^{30}$ reported a significant reduction in internalizing symptoms an after intervention using the SDQ scale with a sample of children living in an orphanage. Taken together, these data demonstrated the effectiveness of the FunFRIENDS program in reducing or preventing internalizing symptoms in clinical settings. However, little is known about its performance when applied at the level of universal prevention in the school environment. 
Table 2. Evaluation of the behavior of the children from both groups regarding the perception of parents and teachers at three different times $\left(\mathrm{T}_{0}\right.$, $\mathrm{T}_{1}$ and $\left.\mathrm{T}_{2}\right)$ based on the Intention to treat analysis 1 (last-observation-carried-forward).

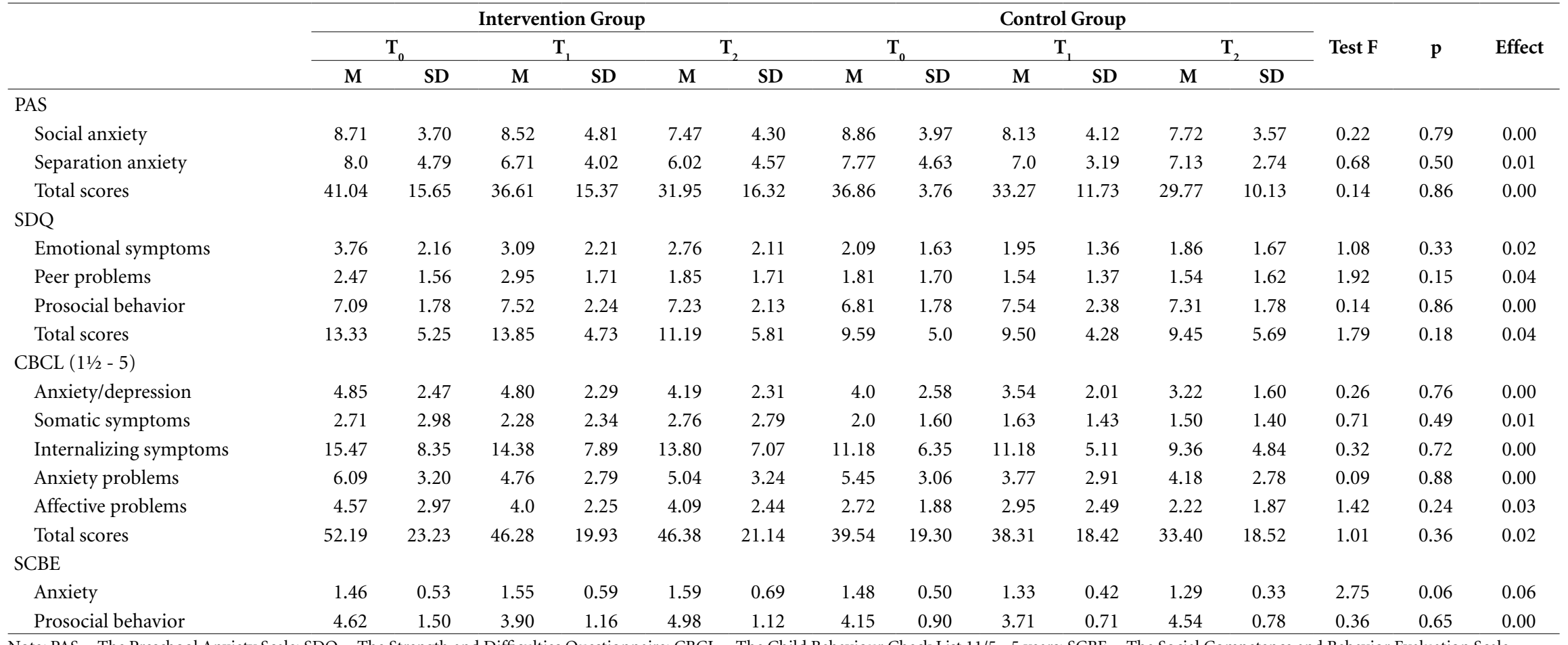

Note: PAS = The Preschool Anxiety Scale; SDQ = The Strength and Difficulties Questionnaire; CBCL = The Child Behaviour Check List 11/5 - 5 years; SCBE = The Social Competence and Behavior Evaluation Scale. 
Table 3. Evaluation of the behavior of the children from both groups regarding the perception of parents and teachers at three different times $\left(\mathrm{T}_{0}\right.$, $\mathrm{T}_{1}$, and $\left.\mathrm{T}_{2}\right)$ based on the Intention to treat analysis 2 (ITT-2).

\begin{tabular}{|c|c|c|c|c|c|c|c|c|c|c|c|c|c|c|c|}
\hline & \multicolumn{6}{|c|}{ Intervention Group } & \multicolumn{6}{|c|}{ Control Group } & \multirow{3}{*}{ Test $\mathrm{F}$} & \multirow{3}{*}{$\mathbf{p}$} & \multirow{3}{*}{ Effect } \\
\hline & \multicolumn{2}{|c|}{$\mathrm{T}_{0}$} & \multicolumn{2}{|c|}{$\mathbf{T}_{1}$} & \multicolumn{2}{|c|}{$T_{2}$} & \multicolumn{2}{|c|}{$\mathbf{T}_{0}$} & \multicolumn{2}{|c|}{$T_{1}$} & \multicolumn{2}{|c|}{$\mathrm{T}_{2}$} & & & \\
\hline & $\mathbf{M}$ & SD & $\mathbf{M}$ & SD & $\mathbf{M}$ & SD & $\mathbf{M}$ & SD & $\mathbf{M}$ & SD & $\mathbf{M}$ & SD & & & \\
\hline \multicolumn{16}{|l|}{ PAS } \\
\hline Social anxiety & 8.71 & 3.70 & 8.07 & 4.56 & 6.87 & 3.79 & 8.86 & 3.97 & 8.35 & 3.56 & 8.17 & 2.71 & 0.61 & 0.54 & 0.01 \\
\hline Separation anxiety & 8.0 & 4.79 & 6.87 & 3.97 & 6.06 & 3.85 & 7.77 & 3.19 & 7.11 & 3.19 & 6.86 & 2.57 & 0.48 & 0.61 & 0.01 \\
\hline Total scores & 41.04 & 15.65 & 36.46 & 15.25 & 29.66 & 11.37 & 36.86 & 13.76 & 33.57 & 10.32 & 29.86 & 7.60 & 0.70 & 0.47 & 0.01 \\
\hline \multicolumn{16}{|l|}{ SDQ } \\
\hline Emotional symptoms & 3.76 & 2.16 & 2.90 & 2.15 & 2.52 & 1.98 & 2.09 & 1.63 & 1.83 & 1.24 & 1.87 & 1.46 & 1.68 & 0.19 & 0.03 \\
\hline Peer problems & 2.47 & 1.56 & 2.94 & 1.68 & 1.78 & 1.62 & 1.81 & 1.70 & 1.63 & 1.31 & 1.45 & 1.19 & 1.49 & 0.23 & 0.03 \\
\hline Prosocial behavior & 7.09 & 1.78 & 7.54 & 2.20 & 7.38 & 2.38 & 6.81 & 1.78 & 7.98 & 1.94 & 7.71 & 1.18 & 0.05 & 0.59 & 0.01 \\
\hline Total scores & 13.33 & 5.25 & 13.90 & 4.13 & 11.30 & 5.41 & 9.59 & 5.0 & 9.17 & 3.84 & 9.48 & 4.77 & 1.85 & 0.17 & 0.04 \\
\hline \multicolumn{16}{|l|}{ CBCL $\left(1 \frac{1 / 2}{-5}\right)$} \\
\hline Anxiety/depression & 4.85 & 2.47 & 4.80 & 2.29 & 4.19 & 2.31 & 4.0 & 2.58 & 4.85 & 2.47 & 3.22 & 1.60 & 0.26 & 0.76 & 0.00 \\
\hline Somatic symptoms & 2.71 & 2.98 & 2.28 & 2.34 & 2.76 & 2.79 & 2.0 & 1.60 & 2.28 & 2.34 & 1.50 & 1.40 & 0.71 & 0.49 & 0.01 \\
\hline Internalizing symptoms & 15.47 & 8.35 & 14.38 & 7.89 & 13.80 & 7.73 & 11.18 & 6.35 & 11.19 & 5.61 & 9.36 & 4.84 & 0.32 & 0.72 & 0.00 \\
\hline Anxiety problem & 6.09 & 3.20 & 4.76 & 2.79 & 4.18 & 2.78 & 5.45 & 3.06 & 3.77 & 2.91 & 4.18 & 2.78 & 0.09 & 0.88 & 0.00 \\
\hline Affective problem & 4.57 & 2.97 & 4.0 & 2.25 & 4.09 & 2.44 & 2.72 & 1.88 & 2.95 & 2.49 & 2.22 & 1.87 & 1.42 & 0.24 & 0.03 \\
\hline Total scores & 52.19 & 23.23 & 46.28 & 19.93 & 46.38 & 21.14 & 39.54 & 19.03 & 38.31 & 18.42 & 33.40 & 18.52 & 1.01 & 0.36 & 0.02 \\
\hline \multicolumn{16}{|l|}{ SCBE } \\
\hline Anxiety & 1.46 & 0.53 & 1.55 & 0.69 & 1.59 & 0.79 & 1.48 & 0.50 & 1.32 & 0.41 & 1.23 & 0.30 & 3.01 & 0.06 & 0.06 \\
\hline Prosocial behavior & 4.62 & 1.50 & 3.95 & 1.16 & 4.98 & 1.12 & 4.15 & 0.90 & 3.72 & 0.67 & 4.63 & 0.69 & 0.33 & 0.65 & 0.00 \\
\hline
\end{tabular}

Note: PAS = The Preschool Anxiety Scale; SDQ = The Strength and Difficulties Questionnaire; CBCL $=$ The Child Behaviour Check List $11 / 5-5$ years; SCBE $=$ The Social Competence and Behavior Evaluation Scale. 


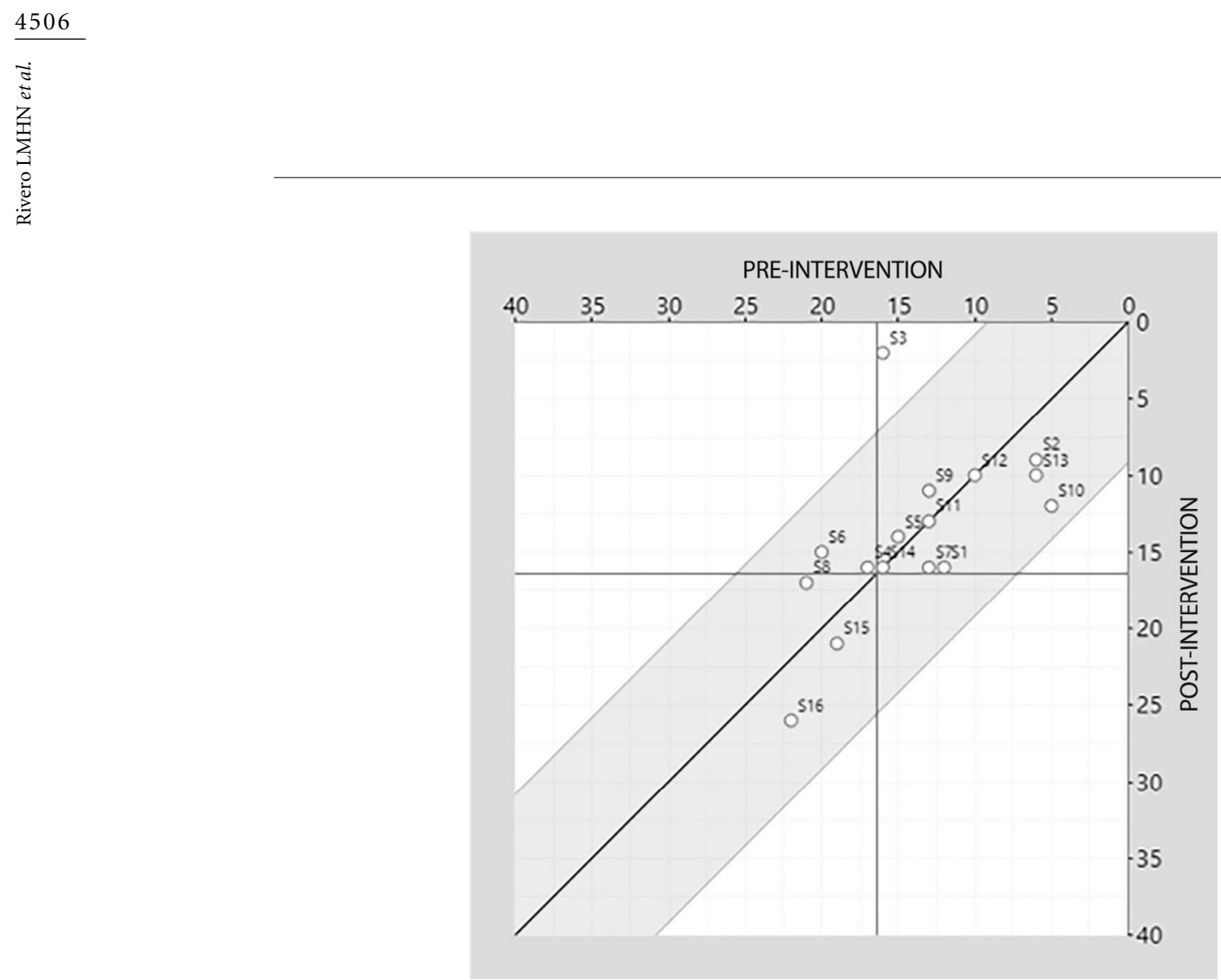

Figure 2. JT analysis of all participants (bullets) from intervention group. Those bullets upper the horizon line indicates adjusted behaviors measured by SDQ instrument.

Regarding the JT analysis, it was detected that the intervention was effective only on two children in which they improved the scores from SDQ instrument. As shown on Figure 2, most of the children started the intervention on the second quadrant indicating they already had adjusted behaviour. We hypothesized that the period from $\mathrm{T} 1$ and $\mathrm{T} 2$ could be insufficient to detect possible alterations on the behaviours indicating the need to evaluate the effect of the program several months later, as some authors have suggested that participants in prevention programs may need to go through a period of high risk for the preventive effect of the intervention to arise ${ }^{31}$.

Many studies are based on clinical trials conducted as part of therapeutic approaches, possibly because populations with high levels of internalizing problems may benefit to a greater extent from this intervention model ${ }^{32}$. It is possible, therefore, that the profile of these children makes it more likely that they will present changes about internalizing problems, unlike in programs focused on universal prevention strategies. In this respect, this type of intervention can present a relatively low dose of intervention and does not offer enough exposure to impact children who are at high risk of a specific disorder ${ }^{33}$.

Thus, while internalizing problems are directly related to the likelihood of mental illness, prosocial behaviours are considered positive measures of universal prevention and promotion in mental health. Prosocial behaviours are any voluntary act designed to benefit another without seeking rewards, being imbued with empathy, generosity, and solidarity ${ }^{34-36}$.

In the present study, it was not detected a significant increase in the frequency of prosocial behaviours. In two reports evaluating prosocial behaviour using the SDQ instrument, there were also no differences between the experimental and control groups ${ }^{26,30}$. However, some authors did observe a significant effect of the FunFRIENDS program on the pro-social behaviors ${ }^{13,15}$. In all of these studies, the prosocial behaviours were measured using the BERS (Behavioural and Emotional Rating Scale) instrument.

Although this was a randomized controlled trial, it has some limitations to be considered. 


\section{Collaborations}

LMHN Rivero, LZP Figueiredo, ALM Andrade and D De Micheli were responsible for the study concept and design. LMHN Rivero and BO Pinheiro contributed to the acquisition of data. All authors assisted with data analysis and the interpretation of findings and provided a critical revision of the manuscript for important intellectual content. All authors reviewed the content and approved the final version.

\section{Funding}

This study was financed in part by the Coordenação de Aperfeiçoamento de Pessoal de Nível Superior - Brasil (CAPES) - Finance Code 001.

\section{Acknowledgment}

The authors are thankful for Sônia Regina Fiorim Enumo, Ph.D. from PUC-Campinas for critical review of the text and Andressa Melina Becker da Silva, Ph.D. from UNIMEP for JT statistical analyses and support on the interpretation of the data. related to the behaviour of the children in the classroom and the leadership style of the teachers.

In summary, we did not find an effect of the FUnFRIENDS to reduce the intensity of internalizing problems and increasing the incidence of pro-social behaviours. These data can be used to adapt specific FunFRIENDS protocols for Brazilian children, taking into account local conditions.

\section{References}

1. Lijster JM, Dierckx B, Utens EMWJ, Verhulst FC, Zieldorff C, Dieleman GC, Legerstee JS. The Age of Onset of Anxiety Disorders: A Meta-analysis. Can J Psychiatry 2017; 62(4):237-246.

2. Fleitlich-Bilyk B, Goodman R. Prevalence of child and adolescent psychiatric disorders in southeast Brazil. J Am Acad Child Adolesc Psychiatry 2004; 43(6):727734.

3. World Health Organization (WHO). Depression and Other Common Mental Disorders Global Health Estimates. Geneva: WHO; 2017.

4. Bandelow B, Michaelis S. Epidemiology of anxiety disorders in the 21st century. Dialogues Clin Neurosci 2015; 17(3):327-335.

5. Finsaas MC, Buffered SJ, Dougherty LR, Carlson GA, Klein DN. Preschool psychiatric disorders: homotypic and heterotypic continuity through middle childhood and early adolescence. Psychol Med 2018; 48(13):2159-2168.

6. Leme VBR, Del Prette ZAP, Koller SH, Del Prette A. Social skills and bioecological model of human development: analyze and perspectives. Psicol Soc 2016; 28(1):181-193

7. Poletto M, Koller SH. Ecological contexts: furthering resilience, risk and protection factors. Estud Psicol 2008; 25(3):405-416.

8. Flett GL, Hewitt PL. Disguised distress in children and adolescents "flying under the radar" why psychological problems are underestimated and how schools must respond. Can J School Psychol 2013; 28(1):12-27.

9. Werner-Seidler A, Perry Y, Calear AL, Newby JM, Christensen H. School-based depression and anxiety prevention programs for young people: A systematic review and meta-analysis. Clin Psychol Rev 2017; 51:30-47.

10. Pahl KM, Barrett PM. The development of socialemotional competence in preschool-aged children An introduction to the fun FRIENDS program. J Psychol Counsellors Schools 2007; 17(1):81-90. 
11. Cooley-Strickland MR, Griffin RS, Darney D, Otte K, Ko J. Urban African American youth exposed to community violence: A school-based anxiety preventive intervention efficacy study. J Prev Interv Community 2011; 39(2):149-166.

12. Essau CA, Conradt J, Sasagawa S, Ollendick TH. Prevention of anxiety symptoms in children: Results from a universal school-based trial. Behav Ther 2012; 43(2):450-464.

13. Pahl KM, Barrett PM. Preventing anxiety and promoting social and emotional strength in preschool children: A universal evaluation of the Fun FRIENDS program. Advances School Mental Health Promotion 2010; 3(3):14-25.

14. Zertuche CP. Effectiveness of the "Amistad y Diversion" program in the development of resilience in preschool children [thesis]. México: Universidad de Monterrey; 2012.

15. Anticich SA, Barrett PM, Silverman W, Lacherez P, Gillies R. The prevention of childhood anxiety and promotion of resilience among preschool-aged children: A universal school-based trial. Advances School Mental Health Promotion 2013; 6(2):93-121.

16. Almeida JP, Viana V. Anxiety in Portuguese pre-school children - adaptation of the spence pre-school anxiety scale. Psicol Saúde Doenças 2013; 14(3):470-483.

17. Silvares EFM, Rocha MM, Linhares MBM. Inventário de Comportamentos de Crianças entre 1 1/2-5 anos (CBCL/11/2-5). Versão brasileira do "Child Behavior

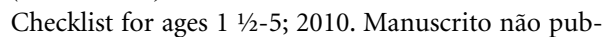
licado.

18. Fleitlich B, Cortázar PG, Goodman R. Strengths and difficulties questionnaire (SDQ). Infanto Rev Neuropsiquiatr Infância Adolesc 2000; 8(1):44-50.

19. Bigras M, Dessen MA. Social competence and behavior evaluation in Brazilian preschoolers. Early Education Development 2002; 13(2):139-152.

20. Andrade ALM, Teixeira LRDS, Zoner CC, Niro NN, Scatena A, Amaral RAD. Factors associated with postpartum depression in social vulnerability women. Rev Eletrônica Saúde Mental Álcool Drog 2017; 13(4):196204.

21. Cruz FAD, Scatena A, Andrade ALM, De Micheli D. Evaluation of Internet addiction and the quality of life of Brazilian adolescents from public and private. Estud Psicol 2018; 35(2):193-204.

22. Cohen J. Statistical power analysis for the behavioral sciences. Hilsdale: Lawrence Earlbaum Associates; 1988.

23. Jacobson NS, Truax P. Clinical significance: a statistical approach to defining meaningful change in psychotherapy research. J Consult Clin Psychol 1991; 59(1):1-12.

24. Aguiar AAR, Aguiar RG, Del Prette ZAP. Calculando a significância clínica e o índice de mudança confiável em pesquisa-intervenção. São Carlos: UFSCAR; 2009.

25. Barrett P, Fisak B, Cooper M. The treatment of anxiety in young children: Results of an open trial of the Fun FRIENDS program. Behav Change 2015; 32(4):231242.
26. Dohl AH. Managing anxiety through childhood social-emotional development [thesis]. Vancouver: The University of British Columbia; 2013.

27. Achenbach TM, Ivanova MY, Rescorla LA, Turner LV, Althoff RR. Internalizing/externalizing problems: Review and recommendations for clinical and research applications. J Am Acad Child Adolesc Psychiatry 2016; 55(8):647-656.

28. Shortt AL, Barrett PM, Fox TL. Evaluating the FRIENDS program: A cognitive-behavioral group treatment for anxious children and their parents. $J$ Clin Child Psychol 2001; 30(4):525-535.

29. Siu AF. Using friends to combat internalizing problems among primary school children in Hong Kong. $J$ Cognitive Behav Psychother 2007; 7:11.

30. Gallegos-Guajardo J, Ruvalcaba-Romero N, Langley A, Villegas-Guinea D. Selective prevention for anxiety and resilience promotion: outcomes of anxiety prevention and resilience program with girls at risk. Pensando Psicol 2015; 11(18):11-23.

31. Gillham JE, Shatté AJ, Reivich K. Needed for prevention research: Long-term follow up and the Evaluation of mediators, moderators, and lay providers. Prevention Treatment 2001; 4(1):article ID 9c.

32. National Research Council. Preventing mental, emotional, and behavioral disorders among young people: Progress and possibilities. Washington: National Academies Press; 2009.

33. Sanchez AL, Cornacchio D, Poznanski B, Golik AM, Chou T, Comer JS. The effectiveness of school-based mental health services for elementary-aged children: a meta-analysis. J Am Acad Child Adolesc Psychiatry 2018; 57(3):153-165.

34. Eisenberg N, Spinrad TL, Taylor ZE, Liew J. Relations of Inhibition and Emotion Related Parenting to Young Children's Prosocial and Vicariously Induced Distress Behavior. Child Dev 2019; 90(3):846-858.

35. Fernandes AO, Oliveira NR. Prosocial Behavior among Adolescents in Institutional Sheltering. Psicol Teoria Pesq 2017; 33(1):e3331.

36. Löhr SS, Pereira ACS, Andrade ALM, Kirchner LDF. Preventive programs evaluation: experience story. Psicol Estud 2007; 12(3):641-649.

Article submitted 16/03/2018

Approved 15/04/2019

Final version submitted 17/04/2019 